\title{
PRÁTICAS SEGURAS PARA ADMINISTRAÇÃO DE MEDICAMENTOS: CONSTRUÇÃO E VALIDAÇÃO DE INSTRUMENTO
}

Mônica Jordão de Souza, Daniel Sundfeld Spiga Real², Isabel Cristina Kowal Olm Cunhal³, Elena Bohomol ${ }^{4}$

Objetivo: : Construir e validar um instrumento de avaliação do processo de administração de medicamentos. Metodologia: Utilizou-se um desenho não experimental e a sequência da pesquisa metodológica, ou seja, definição do construto, formulação dos itens, avaliação da confiabilidade e avaliação da validade de conteúdo. Resultado: Obteve-se um instrumento com 54 itens divididos em 10 áreas, com nível de concordância entre 90 a 100\% e Alpha de Cronbach de 0,94. Conclusão: Pode afirmar-se que o instrumento final é um material de qualidade, que permite o monitoramento dos processos de trabalho que interferem na ocorrência de erros de administração de medicamentos.

Descritores: Erros de Medicação; Sistemas de Medicação; Segurança do Paciente; Estudos de Avaliação.

\section{SAFE PRACTICES FOR MEDICATION ADMINISTRATION: INSTRUMENT CONSTRUCTION AND VALIDATION.}

Construct and validate an instrument for evaluating the medication administration process. Method: We used a nonexperimental design and the sequence of the methodological research, that is, the definition of the construct, formulation of the items, evaluation of reliability and evaluation of the content validity. Result: An instrument was obtained with 54 items divided into 10 areas, with a level of agreement between 90 and 100\% and Cronbach's Alpha of 0.94. Conclusion: It can be stated that the final instrument is a quality material, which allows the monitoring of work processes that interfere in the occurrence of medication administration errors.

Descriptors: Medication Errors, Medication Systems, Patient Safety, Evaluation Studies.

\section{PRÁCTICAS SEGURAS PARA LAADMINISTRACIÓN DE DROGAS: CONSTRUCCIÓN Y VALIDACIÓN DE INSTRUMENTOS.}

Objetivo: Construir y validar un instrumento de evaluación del proceso de administración de medicamentos. Método: Se utilizó un diseño no experimental y la secuencia de la metodología de búsqueda, o construir formulación definición de artículos, la evaluación fiabilidad y la evaluación de la validez del contenido. Resultado: Se obtiene un instrumento con 54 ítems divididos en 10 zonas, con el grado de concordancia entre el 90-100\% y Alpha de Cronbach de 0,94. Conclusión: Se puede decir que el instrumento final es un material de calidad, lo que permite el seguimiento de los procesos de trabajo que influyen en la aparición de errores de administración de medicamentos.

Descriptores: Errores de Medicación; Sistemas de Medicación; Seguridad del Paciente; Estudios de Evaluación 


\section{INTRODUÇÂO}

Agestão do sistema de medicação pelos estabelecimentos de assistência à saúde (EAS) é de relevância ímpar, sobretudo quando se sabe que em alguns países o gasto anual com hospitalizações adicionais, litígio, entre outros, decorrentes dos erros de medicação (EM) é da ordem de US\$ 6 a US\$ 29 bilhões $^{(1)}$. Esses dados tornam evidentes, não somente o impacto financeiro e a depreciação da imagem dos EAS, como também as possiveis repercussões causadas a cada paciente e família lesada.

Frente a essas situações e considerando a complexidade do sistema de medicação, bem como o expressivo número de profissionais envolvidos em seus processos (organização e gerenciamento, seleção e aquisição, estoque, prescrição e transcrição, preparo e dispensação, e administração e monitoramento), (2) o que se tem percebido é o grande interesse de pesquisadores em mensurar os EM. Assim, é de extrema importância que cada processo seja avaliado e se compreendam as fontes de risco e possíveis estratégias de mitigação dos erros.

O processo em que há maior participação da equipe de enfermagem é o de administração de medicamentos e, por isso, destaca-se a imprescindibilidade do enfermeiro, como gestor do cuidado, conhecer políticas públicas voltadas à segurança do paciente, medidas de prevenção e redução de erros e custos. Vale lembrar também que fazer a gestão do sistema de medicação requer planejamento e avaliação contínua da qualidade para que todos os pacientes tenham resguardado seu direito a não sofrer danos.

Essa tem sido a lógica dos Programas de Acreditação, como da Joint Commission International, da Accreditation Canada International e da Organização Nacional de Acreditação que, por meio de padrões bem definidos, avaliam e certificam a qualidade das instituições de saúde, aplicando programas que estimulam a transformação da realidade. ${ }^{(3)}$

Todavia, ainda que se conheçam os benefícios desses programas, suas contratações não podem ser realizadas por muitos EAS nacionais devido a restrições orçamentárias. Outro aspecto que dificulta a adesão a esses programas está no fato de que alguns deles foram construídos considerando padrões de saúde internacionais e não contemplam todas as nuances e peculiaridades da realidade brasileira, sobretudo no que diz respeito ao sistema de medicação.
Portanto, esse estudo teve como objetivo a construção e validação de um instrumento de avaliação do processo de administração de medicamentos, baseado no "Protocolo de segurança da prescrição, uso e administração de medicamentos"(4), do Programa Nacional de Segurança do Paciente (PNSP).

\section{METODOLOGIA}

Trata-se de um estudo com desenho não experimental, tipo pesquisa metodológica e aprovado pelo Comitê de Ética em Pesquisa da Universidade Federal de São Paulo sob o no 554.046 e CAEE: 27845114.3.0000.5505. Todos os participantes receberam um Termo de Consentimento Livre e Esclarecido (TCLE).

Para o desenvolvimento do instrumento foram utilizadas as seguintes fases: definição do construto, formulação dos itens, avaliação da confiabilidade e avaliação da validade de conteúdo.(5) Como estratégia para definição do construto, foi realizada leitura crítica do "Protocolo de segurança da prescrição, uso e administração de medicamentos" (4), cuja estrutura consiste em sete grandes tópicos, dos quais três se relacionam às práticas seguras para os processos de prescrição, distribuição e administração de medicamentos, sendo este último o objeto de interesse de presente estudo.

Para a formulação dos itens, foram selecionadas do protocolo as recomendações de práticas seguras de administração de medicamentos. Elas foram transcritas, reelaboradas e organizadas até a estruturação de um instrumento que foi enviado, por meio eletrônico, para julgamento.

Para a aferição da validade de conteúdo, utilizou-se a porcentagem de concordância (6) entre os painelistas, estabelecendo um consenso superior a $90 \%$ para aceitação. O grupo de painelistas convidado foi selecionado pelo site da Plataforma Lattes e por indicações de membros da Agência Nacional de Vigilância Sanitária (ANVISA) e de gestores de qualidade de hospitais públicos, privados e da atenção básica, com formação multidisciplinar e com experiência no sistema de medicação.

Para a confiabilidade da consistência interna, utilizou-se o coeficiente Alfa de Cronbach, estabelecendo-se como valor adequado um coeficiente $\geq 0,75$. Os cálculos estatísticos foram realizados com o auxilio do programa STATISTICA $12.0 . .^{(7)}$ 


\section{RESULTADOS}

O instrumento enviado aos painelistas se estruturou em 54 itens divididos em 10 áreas, com as recomendações de práticas seguras de administração de medicamentos. Cada área teve sua constituição baseada em afirmativas que foram avaliadas pelos painelistas, conforme a Tabela 1

Tabela 1. Área, número de itens e intervalo da porcentagem de concordância. Brasil, 2017.

\begin{tabular}{|c|c|c|c|}
\hline \multirow{2}{*}{ Área } & \multirow{2}{*}{$\begin{array}{l}\text { № de } \\
\text { Itens }\end{array}$} & \multicolumn{2}{|c|}{ Porcentagem de Concordânia } \\
\hline & & Menor Valor & Maior Valor \\
\hline Paciente certo & 5 & $92,5 \%$ & $100 \%$ \\
\hline Forma certa & 4 & $97,5 \%$ & $100 \%$ \\
\hline Resposta certa & 3 & $92,5 \%$ & $100 \%$ \\
\hline Medicamento certo & 3 & $97,5 \%$ & $100 \%$ \\
\hline Via certa & 4 & $97,5 \%$ & $100 \%$ \\
\hline Hora certa & 5 & $90,0 \%$ & $100 \%$ \\
\hline Dose certa & 4 & $97,5 \%$ & $100 \%$ \\
\hline Registro certo & 13 & $92,5 \%$ & $100 \%$ \\
\hline Orientação certa & 4 & $97,5 \%$ & $100 \%$ \\
\hline
\end{tabular}

Dos 118 convidados, 40 (33,9\%) fizeram parte da amostra. Do restante, 52 (44,1\%) não responderam ao convite, 16 $(13,5 \%)$ se recusaram a participar e 10 (8,5\%) responderam que participariam do estudo, porém, como não enviaram o TCLE assinado, foram excluídos.

Como caracterização da amostra, a maioria foi do sexo feminino (34; 85,0\%), com médias de: idade de 43,3 anos; tempo de formado de 19,9 anos e tempo de experiência no sistema de medicação de 16,3 anos. Quanto à formação, 22 (55,0\%) tinham graduação em enfermagem, sete $(17,5 \%)$ em farmácia, seis $(15,0 \%)$ em medicina, quatro

$(10,0 \%)$ em outras profissões da área da saúde (biomedicina, fisioterapia, nutrição e odontologia) e um (2,5\%) com graduação em farmácia e enfermagem.

Em relação ao local de trabalho, 31 (77,5\%) eram do Estado de São Paulo, quatro do Distrito Federal (10,0\%), três de Minas Gerais (7,5\%) e dois do Rio de Janeiro (2,5\%).

No tocante à natureza do trabalho, 20 (50,0\%) eram de instituições públicas, 18 (45,0\%) de instituições privadas, um $(2,5 \%)$ de instituição filantrópica e um (2,5\%) de instituição mista (público-privada).

A experiência no sistema de medicação também foi investigada, de modo que cada painelista poderia ter mais de um tipo de experiência no sistema de medicação. Observouse uma grande variedade de áreas de atuação, sendo que a maior delas foi na assistência (32; 80,0\%), seguida da gerência/ gestão (25; 62,7\%), avaliação de serviços (11; 27,5\%), docência (6; 15,0\%), pesquisa (3; 7,5\%), formulação de políticas públicas (1; 2,5\%) e identificação e avaliação de incidentes e eventos adversos relacionados ao sistema de medicação (1; 2,5\%).

Como o nível de concordância entre os painelistas variou de 90 a 100\% e a confiabilidade da consistência interna se demonstrou elevada com o resultado do Alpha de Cronbach de 0,94 , o instrumento final se manteve com os 54 itens subdivididos em 10 subáreas, sem necessidade de modificação (Figura 1).

\section{DISCUSSÃO}

Conhecer e saber identificar padrões de segurança relativos ao processo de administração de medicamentos é uma ação gerencial que tem o potencial de desencadear ações que mitiguem e previnam erros. Pesquisa cujo objetivo era determinar a frequência dos EM no departamento de emergência de um hospital de ensino terciário, identificou que $96,5 \%$ dos pacientes estudados experimentaram pelo menos um EM e uma taxa de 3,5 erros por paciente. A maioria deles ocorreu durante o processo de administração $(37,6 \%)$, seguido do de prescrição (21,1\%) e transcrição (10,0\%). ${ }^{(8)}$

Estudo descritivo e observacional direto sobre erros de administração de medicamentos no Hospital Universitário de Ain Shams, por um periodo de três meses, observou um total de 5531 erros, com um número médio de erros de 2,67 por observação. Mais de $85 \%$ das observações tiveram pelo menos um erro e a taxa de erro global foi de 37,68\% (por 100 oportunidades de erro). A maior taxa de erro foi detectada em injeções, especialmente na via intravenosa (39,58\%) e dentre os erros mais frequentes estava a técnica errada (78,90\%). ${ }^{(9)}$ Esses dados, além de alarmantes, evidenciam a importância da proposição de práticas seguras e do uso de instrumentos de avaliação capazes de fornecer informações precisas sobre o processo de administração de medicamentos nos EAS.

Como instrumento de avaliação das práticas de segurança do sistema de medicação, o que se encontra na literatura disponivel para consulta e utilização é um material do Institute for Safe Medication Practices (ISMP) em parceria com a American Hospital Association e o Health Research and Educational Trust. (10) Intitulado "2011 ISMP Medication Safety Self Assessment for Hospitals" esse material, publicado em língua inglesa e desenvolvido para ser utilizado por instituições hospitalares, contém 270 itens de avaliação do sistema de medicação divididos em 10 partes. ${ }^{(11)}$

No entanto, a despeito de ser um instrumento de livre acesso e bastante utilizado internacionalmente, não foi traduzido e adaptado para a língua portuguesa. Além disso, foi desenvolvido para aplicação apenas em instituições hospitalares e apresenta recomendações que não 
representam as diversas peculiaridades da vasta realidade brasileira.

Nesse escopo, o instrumento do presente estudo se faz conveniente por contemplar itens atualizados e contextualizados às exigências do Ministério da Saúde e da ANVISA como também dos conselhos de classe dos profissionais de saúde, servindo como importante ferramenta de gestão para avaliar a segurança do processo de administração de medicamentos das instituições de saúde nacionais. Outro fator que confere vulto ao produto desse estudo, quando comparado ao material do ISMP, é o seu tamanho, uma vez que uma grande quantidade de itens pode ser fator limitante à qualidade dos dados coletados, principalmente por exigir muita disposição, dedicação, envolvimento com o trabalho e disponibilidade de tempo para sua aplicação.

Outrossim, as questões validadas do instrumento final demonstram sua capacidade de utilização em qualquer EAS que utilize "medicamentos para profilaxia, exames diagnósticos, tratamento e medidas paliativas"(4) Exemplo disso pode ser verificado no item 6.3 ("Adequam-se os horários de administração dos medicamentos à rotina de uso já estabelecida pelo paciente antes da internação, sempre que possível") que enfoca uma prática segura a ser aplicada em instituições hospitalares, por exemplo; e no item 9.2 ("Nas prescrições ambulatoriais, são registradas todas as orientações sobre como utilizar o medicamento, bem como as recomendações não farmacológicas") que aborda aspectos específicos dos serviços ambulatoriais.

Adicional exemplo, desta vez destacando o acesso aos recursos que cada EAS tem, pode citar-se as seguintes questões validadas: "Certifica-se de que a infusão programada é a prescrita para aquele paciente" e "Padronizam-se equipamentos como bombas de infusão, limitando a variedade de opções." Nesse caso há uma recomendação genérica para os estabelecimentos que não contam com bombas de infusão e uma para aqueles que dispõem desses equipamentos.

Somado a isso, nosso instrumento orienta ações seguras a serem seguidas em relação ao chamado "Nove certos" da administração de medicamentos, ou seja: medicamento certo, paciente certo, dose certa, via certa, hora certa, registro certo, ação certa, forma certa e orientação/monitoramento certo.(4) Trata-se de um preceito clássico considerado o pilar do ensino sobre práticas seguras da administração de medicamentos nos cursos de enfermagem e que, originariamente, era denominado de "Cinco Certos" (paciente certo, medicamento certo, dose certa, via certa e hora certa). Contudo, é importante salientar que se os "Nove Certos" não estiverem ancorados em políticas e procedimentos institucionais organizados e bem implantados podem dar uma falsa impressão de segurança. ${ }^{(12)}$
Um aspecto que também deve ser enfatizado, além da distribuição dos itens em áreas que correspondem aos "Nove Certos", é o fato de haver uma área, denominada "Outras Recomendações", que contempla atividades diversas e relacionadas à gestão dos EAS, mas que se não realizadas podem impactar na segurança do processo de administração de medicamentos como um todo. Ademais, ressalva-se que a quantidade de itens por área não indica grau de importância, nem possui significância comparativa entre elas.

Outro aspecto que confere robustez ao nosso instrumento é a inserção de uma "Legenda" com critérios de avaliação (A. Nenhuma atividade implementada; B. Atividade considerada, mas não implementada; C. Atividade parcialmente implementada em algumas ou todas as áreas; D. Atividade completamente implementada em algumas áreas; E. Atividade completamente implementada em todas as áreas; F. Não se aplica) que foi acrescentada ao instrumento final para que, ao ser utilizado pelos EAS, o aplicador consiga definir em qual escala a atividade está implementada.

Sabendo que a subjetividade é inerente aos processos de avaliação e que sem sua minimização, existe grande chance de formulação de pareceres repletos de juízos de valor, o estabelecimento de critérios bem definidos de avaliação contribui para a redução do fator subjetivo das aplicações, diferindo o presente instrumento dos utilizados pelos Programas de Acreditação.

No tangente à composição do grupo que julgou os itens que compunham o instrumento de avaliação, há que se ressaltar, além do número expressivo de participantes, que sua composição impacta consideravelmente nos resultados obtidos. A heterogeneidade é um ponto bastante discutido na literatura por proporcionar opiniões diversas sobre um mesmo tema ${ }^{(13)}$ e, por isso, nesse estudo, optou-se, por uma amostra composta por indivíduos de diferentes formações. $\mathrm{Na}$ assistência à saúde, é de suma importância o trabalho em equipe multiprofissional para que os objetivos estratégicos e suas metas sejam alcançados e garantam uma assistência de qualidade dentro do tripé de avaliação proposto por Donabedian (estrutura, processo e resultado).

Em relação à idade, tempo de formado e tempo de experiência no sistema de medicação, as médias obtidas refletem grande experiência profissional e de vida dos painelistas. Isso permite afirmar que eles apresentavam know-how para julgar, reduzindo as chances de vieses e garantindo a relevância dos itens que representam ações seguras na administração de medicamentos.

Um estudo que desenvolveu, mediante a opinião de especialistas, um conjunto de variáveis baseados nos princípios da Política Nacional de Humanização também utilizou um painel multidisciplinar e, à semelhança do que 
encontramos, a maioria dos respondentes era do sexo feminino e enfermeiras. $O$ dado apresentado nesse estudo que difere do encontrado em atual trabalho foi a titulação, já que eles tiveram maioria de indivíduos com doutorado, seguido de mestrado e especialização.(14)

Quanto à natureza da instituição de trabalho dos participantes, percebe-se que houve uma distribuição equilibrada entre público e privado. Embora existam algumas semelhanças entre esses serviços, como as respostas ao desperdício, os incentivos ao desempenho e o foco na eficiência, o que se nota é uma grande disparidade entre eles. (15) Nas instituições públicas, por exemplo, observa-se amplo controle sobre a autonomia administrativa e financeira no que diz respeito à aquisição de bens e serviços, conferindo morosidade aos processos de compra. Por outro lado, nas instituições privadas há maior facilidade na incorporação de novas tecnologias que impactam diretamente nos objetivos institucionais.(15) Dessa forma, contar com experts com vivências ambivalentes garantiu percepções sobre realidades diferentes, com disponibilização de recursos também diferentes.

Aspecto adicional que contribuiu para a qualidade do processo de construção e validação do presente instrumento foi a diversidade de experiência dos profissionais, garantindo que saberes se complementassem. A experiência no ensino, pesquisa, formulação de políticas públicas e identificação e avaliação de incidentes e EA relacionados ao sistema de medicação permitiram que os itens fossem julgados considerando um conhecimento acumulado. Isso significa dizer que a bagagem teórico-prática dos painelistas contribuiu para a construção de um instrumento com potencialidade de diagnosticar situações e estimular a busca por ações de melhoria a partir de tomadas de decisões assertivas.

A despeito de um número menor quando comparado à assistência e gerência/gestão, havia indivíduos com experiência em avaliação de serviços. Ser avaliador requer competências específicas e um treinamento intensivo independente da formação acadêmica. Profissionais dessa área tem grande conhecimento sobre a dinâmica de instituições de saúde de diferentes portes, níveis e natureza. Isso emoldura um opinar considerando seus saberes prévios em utilização de diferentes instrumentos de avaliação.

Em relação à consistência interna, sabe-se que a literatura estabelece como confiabilidade "muito alta" valores de Alpha de Cronbach maior ou igual a 0,9,(16) nivel alcançado em presente trabalho. Isso permite, somado ao elevado nível de concordância entre os painelistas, afirmar que o instrumento carrega consigo a viabilidade de ser aplicado nos mais diversos cenários do sistema de saúde brasileiro, bem como possuir as prerrogativas para demais validações.

Como limitação do estudo é importante ressaltar que esse trabalho apresenta apenas a validação de conteúdo e a avaliação da consistência interna do instrumento, necessitando de outras validações para sua completa implementação.

\section{CONCLUSÃO}

Não há na literatura nenhum instrumento validado em língua portuguesa e que seja especifico para a avaliação da segurança do processo de administração de medicamentos como o proposto no presente trabalho. O produto final desse estudo considera os princípios de utilidade, oportunidade, factibilidade, confiabilidade, objetividade e direcionalidade(15) para que possa ser utilizado pelos EAS brasileiros de forma autoaplicável, permitindo o monitoramento e implantação de melhorias nos processos de trabalho que interferem diretamente na ocorrência de erros de administração de medicamentos. 


\section{REFERÊNCIAS}

1. Cousins DH, Gerrett D, Warner B. A review of medication incidents reported to the National Reporting and Learning System in England and Wales over 6 years (2005-2010). Br J Clin Pharmacol. 2012 Oct:74(4):597-604.

2. Joint Commission International. Padrões de acreditação da Joint Comission International para Hospitais [Internet]. 4th ed. Rio de Janeiro: Consórcio Brasileiro de Acreditação (CBA); 2010 [cited 2017 Jan 29]. 296 p. Available from: https://www.jcrinc.com/assets/1/14/ EBIAS400P_Sample_Pages.pdf.

3. Berssaneti FT, Saut AM, Barakat MF, Calarge FA. Existe uma relação entre os programas de acreditação e os modelos de excelência organizacional?. Rev esc enferm USP [Internet]. 2016 [cited 2017 Fev 13]; 50(4):650-57. Available from: http://www.scielo.br/pdf/reeusp/v50n4/ pt_0080-6234-reeusp-50-04-0650.pdf

4. Agência Nacional de Vigilância Sanitária. Protocolo de segurança na prescrição, uso e administração de medicamentos [Internet]. Brasilia: Agência Nacional de Vigilância Sanitária 2013 [cited 2017 Jan 29]. 45 p. Available from: http://www20.anvisa.gov.br/segurancadopaciente/ index.php/publicacoes/item/seguranca-na-prescricao-uso-e-administracao-de-medicamentos.

5. LoBiondo-Wood G, Haber J. Desenhos não-experimentais. In: LoBiondo-Wood G, Haber J, editors. Pesquisa em enfermagem: métodos, avaliação crítica e utilização. 4a ed. Rio de Janeiro: Guanabara-Koogan; 2001. p. 110-21.

6. Alexandre NMC, Coluci MZO. Validade de conteúdo nos processos de construção e adaptação de instrumentos de medidas. Ciênc Saúde Coletiva [Internet]. 2011 [cited 2017 Jan 31];16(7):3061-68. Available from: http://www.scielo.br/pdf/csc/v16n7/06.pdf

7. Statistica [computer program]. Version 12.0. Tulsa (OK): StatSoft Inc.; 2013

8. Vazin A, Zamani Z, Hatam N. Frequency of medication errors in an emergency department of a large teaching hospital in southern Iran.
Drug Healthc Patient Saf. 2014;6:179-84

9. al Tehewy M, Fahim H, Gad NI, El Gafary M, Rahman SA. Medication Administration Errors in a University Hospital. J Patient Saf. 2016 Mar;12(1):34-9.

10. Institute for Safe Medication Practices. ISMP Self-Assessments [Internet]. Horsham (PA): Institute for Safe Medication Practices: c2015 [cited 2017 Jan 31]. [about 1 screen]. Available from: http://www. ismp.org/selfassessments/default.asp.

11. Institute for Safe Medication Practices. ISMP Medication Safety Self Assessment® for Hospitals [Internet]. Horsham (PA): Institute for Safe Medication Practices; 2011 [cited 2017 Jan 31]. 55 p. Available from: http://www.ismp.org/selfassessments/hospital/201l/full.pdf . 12. Watcher RM. Compreendendo a Segurança do Paciente. 2ed. São Paulo: Artmed; 2013.500p.

13. Pereira RD, Alvim NA. Delphi technique in dialogue with nurses on acupuncture as a proposed nursing intervention. Esc Anna Nery [Internet]. 2015 [cited 2017 Jan 30];19(1):174-80. Available from: http:// www.scielo.br/pdf/ean/v19nl/en_1414-8145-ean-19-01-0174.pdf.

14. Souza DJ, D'Innocenzo M. [Development of the humanization index of health services: step delphi]. Rev Enferm UFPE on line [Internet]. 2014 Jul [cited 2017 Jan 30];8(Supll 1):2365-74. Portuguese. Available from: http://www.revista.ufpe.br/revistaenfermagem/index.php/revista/article/view/6154/pdf_5684

15. Tanaka OY, Tamaki EM. [The role of evaluation in decision-making in the management of health services]. Ciênc Saúde Coletiva [Internet]. 2012 [cited 2017 Jan 31]:17(4):821-8. Portuguese. Available from: http:// www.scielo.br/pdf/csc/v17n4/v17n4aO2.pdf.

16. Freitas AL, Rodrigues SG. A avaliação da confiabilidade de questionários: uma análise utilizando o coeficiente alfa de Cronbach. Anais do 12으 SIMPEP Simpósio de Engenharia de Produção; 2005 Nov 7-9. Bauru, SP [Internet]. 2005 [cited 2017 Jan 31]. [13 p.]. Available from: http://www.simpep.feb.unesp.br/anais_simpep_aux.php?e=12 